\title{
Genome-Based Analysis of a Sequence Type 1049 Hypervirulent Klebsiella pneumoniae Causing Bacteremic Neck Abscess
}

\section{OPEN ACCESS}

Edited by
Yang Wang

China Agricultural University, China

Reviewed by:

Fupin $\mathrm{Hu}$,

Fudan University, China

Haijian Zhou,

National Institute for Communicable

Disease Control and Prevention

(China CDC), China

*Correspondence:

Yunsong Yu

yvys119@zju.edu.cn

Jiancang Zhou

jiancangzhou@zju.edu.cn

tThese authors have contributed equally to this work

${ }^{\ddagger}$ Present address: Qiucheng Shi,

Department of Clinical Laboratory,

The Children's Hospital, Zhejiang

University School of Medicine,

National Clinical Research Center for

Child Health, Hangzhou, China

Specialty section:

This article was submitted to

Infectious Diseases,

a section of the journal

Frontiers in Microbiology

Received: 15 October 2020 Accepted: 24 December 2020

Published: 18 January 2021

Citation:

Lan P, Zhao D, Gu J, Shi Q,

Yan $R$, Jiang $Y$, Zhou J and Yu $Y$ (2021) Genome-Based Analysis of a

Sequence Type 1049 Hypervirulent Klebsiella pneumoniae Causing

Bacteremic Neck Abscess.

Front. Microbiol. 11:617651.

doi: 10.3389/fmicb.2020.617651
Peng Lan 1,2,3t, Dongdong Zhao'1t, Jiong Gu4, Qiucheng Shi2f, Rushuang Yan ${ }^{2,3}$, Yan Jiang ${ }^{2}$, Jiancang Zhou ${ }^{3 *}$ and Yunsong Yu ${ }^{1,2 *}$

1 Department of Infectious Diseases, Sir Run Run Shaw Hospital, Zhejiang University School of Medicine, Hangzhou, China, ${ }^{2}$ Key Laboratory of Microbial Technology and Bioinformatics of Zhejiang Province, Hangzhou, China, ${ }^{3}$ Department of Critical Care Medicine, Sir Run Run Shaw Hospital, Zhejiang University School of Medicine, Hangzhou, China, ${ }^{4}$ Department of Infectious Diseases, The First Hospital of Jiaxing, Zhejiang, China

Hypervirulent Klebsiella pneumoniae (hvKP) has raised grave concerns in recent years and can cause severe infections with diverse anatomic locations including liver abscess, meningitis, and endophthalmitis. However, there is limited data about neck abscess caused by hvKP. A K. pneumoniae strain Kp_whw was isolated from neck abscess. We characterized the genetic background, virulence determinates of the strain by genomic analysis and dertermined the virulence level by serum resistance assay. Kp_whw belonged to sequence type (ST) $1049 \mathrm{~K}$ locus (KL) 5. Kp_whw showed hypermucoviscosity phenotype and was resistant to ampicillin but susceptible to the majority of the other antimicrobial agents. A pLVPK-like virulence plasmid and a chromosomal ICEKp5-like mobile genetic element were carried by Kp_whw, resulting in the risk of dissemination of hypervirulence. The strain exhibited relative higher level of core genome allelic diversity than accessory genome profile, in comparison to hvKP of $\mathrm{K} 1 / \mathrm{K} 2$ serotype. Kp_whw was finally demonstrated as virulent as the ST23 K1 serotype hvKP strain NTUH-K2044 in vitro. In conclusion, this work elaborates the genetic background of a clinical hvKP strain with an uncommon ST, reinforcing our understanding of virulence mechanisms of hvKP.

Keywords: hypervirulent, Klebsiella pneumoniae, neck abscess, ST1049, comparative genomic analysis

\section{INTRODUCTION}

Hypervirulent Klebsiella pneumoniae (hvKP) has raised grave concerns in recent years due to its extraordinary invasiveness and hypervirulence, resulting in considerable risks for morbidity and mortality (Shon et al., 2013). Asia is the epidemic area for hvKP. K1/K2 accounted for $9.8 \%$ of all K. pneumoniae isolates in stools from healthy Chinese individuals in Asia countries (Lin et al., 2012). In recent years, hvKP has been increasingly reported in other continents. The estimated prevalence of hvKP in Canada and the United States was 8.2 and 6.3\%, respectively (Peirano et al., 2013; Chou et al., 2016). According to different areas, the mortality of hvKP infections ranged from 29.2 to 55.1\% (Lin Y.T. et al., 2010; Rafat et al., 2018; Namikawa et al., 2019). hvKp infection is often manifested as liver abscess (Liu et al., 1986; Siu et al., 2012; Joob and Wiwanitkit, 2017). 
However, extrahepatic infections have been described for variable anatomic locations including endoophthalmitis (Liu et al., 1986), osteomyelitis (Prokesch et al., 2016) and meningitis (Melot et al., 2016). Originated in the Asian Pacific Rim in 1980s, hvKP has disseminated worldwide.

A number of virulence factors contribute to the pathogenicity of hvKP including lipopolysaccharide (LPS), capsule, fimbriae, siderophores, and virulence plasmids (Paczosa and Mecsas, 2016; Russo and Marr, 2019). Traditionally, hypermucoviscosity, resulted by over expression of capsular polysaccharides, was considered as a critical characteristic for hypervirulence (Catalan-Najera et al., 2017), as the mucoviscous shield significantly increased the resistance to immunological recognition and killing from the host (Lin et al., 2006; Lin J.C. et al., 2010). Recently, virulence plasmids were proved to have potential to transmit virulence genes among K. pneumoniae strains (Yang et al., 2019). Virulence plasmids encode two siderophores, aerobactin, and salmochelin, and RmpA (regulator of the mucoid phenotype). The virulence plasmid pK2044 harbored by K1 serotype strain NTUHK2044 and pLVPK harbored by K2 serotype strain CG43 were well characterized and were reported associated with invasive syndrome. Besides, virulence genes located in chromosome can also be transmitted via integrative and conjugative element (ICE) (Lam et al., 2018). Virulence plasmids and ICEKps both harbor virulence loci encoding variable virulence factors and are involved in horizontal gene transfer (HGT), conferring the global dissemination of hypervirulence.

Clonal complex 23 (CC23) and some selected serotypes (e.g., $\mathrm{K} 1$ and $\mathrm{K} 2$ ) are commonly deemed as hypervirulent clones (Struve et al., 2015). Most of hvKP strains of K1 serotype belong to CC23, while K2 serotype strains are genetically more diverse and belong to multiple distinct multilocus sequencing types (MLSTs) (Struve et al., 2015). More than a half of severe K. pneumoniae infections such as bacteremia, liver abscess, and invasive extrahepatic infections are caused by $\mathrm{K} 1 / \mathrm{K} 2$ serotype strains (Lin Y.T. et al., 2010). In addition, strains of serotype K5, K20, and K54 are also associated with hypervirulence phenotype (Wyres et al., 2020).

Clinically, hvKP usually causes liver abscess with extrahepatic complications including necrotizing fasciitis, bloodstream infection, meningitis, and endophthalmitis (Siu et al., 2012). Patients infected with hvKP are frequently relative healthy and immunosufficient. However, diabetes is a critical risk factor for hvKP infection, partly due to the suppression of the innate immune system (Alba-Loureiro et al., 2007; Hodgson et al., 2015).

Here, we described a potential hypervirulent $K$. pneumoniae strain belonging to ST1049, an uncommon ST, isolated from neck abscess. K. pneumoniae of ST1049 was previously reported to cause liver abscess and meningitis, and resulting in poor clinical outcomes (Ku et al., 2017; Zhang et al., 2019). However, no genetic evidence was showed that this infrequent ST was a hypervirulent clone. Hence, we will characterize the strain based on genetic background and virulence profile and thus to refresh our knowledge about hvKP pathogenicity.

\section{MATERIALS AND METHODS}

\section{Isolations}

The strain was isolated from a male patient diagnosed with neck abscess in 2018. It was identified to the species level via matrix-assisted laser desorption/ionization mass spectrometry and named as Kp_whw. The antimicrobial susceptibilities of the strain were determined by a VITEK-2 compact system and interpreted according to the M100-S26 guideline established by Clinical and Laboratory Standards Institute (CLSI). Since the clinical characteristics were extracted from the electronic record system and were de-identified, informed consent was waived. In addition, 92 hvKP strains of $\mathrm{K} 1 / \mathrm{K} 2$ serotype from our previous study (Lan et al., 2020) plus NTUH-K2044 (Wu et al., 2009) and hvKP1 (Russo and Gill, 2013) were employed for comparative genomic analysis.

\section{Whole Genome Sequencing}

The strain was cultured to the mid-logarithmic phase in LB broth at $37^{\circ} \mathrm{C}$. Genomic DNA was extracted using the QIAamp DNA Minikit (QIAGEN, Hilden, Germany) and was further purified using the PowerClean DNA cleanup kit (Mo Bio Laboratories, Carlsbad, United States), following the manufacturer's recommendations. The genome was sequenced on an Illumina HiSeq X Ten platform (Illumina, San Diego, United States) using a paired-end $2 \times 150$-base pair protocol by Tianke Company (Hangzhou, China). Derived short reads were de novo assembled using CLC Genomics Workbench 9.5.1 software. The sequences assemblies reported in this paper have been deposited in the European Nucleotide Archive database (accession nos. PRJEB38367 and PRJEB34922). The NCBI accession number for NTUH-K2044 and hvKP1 are AP006725 and AOIZ00000000.

\section{Typing and Detection of Virulence Determinants}

The virulence genes and wzi (a part of K-locus) alleles were identified using Institut Pasteur ${ }^{1}$. Multilocus sequence typing (MLST) analysis for sequence types of $K$. pneumoniae used Institut Pasteur MLST ${ }^{2}$. For MLST, 7 target genes (gapA, infB, $m d h, p g i, p h o E, r p o B$, and $\operatorname{ton} B$ ) were analyzed. To detect the virulence plasmid, the contigs of strain $\mathrm{KP}_{-}$whw were aligned with well-characterized virulence plasmid pLVPK (accession no. AY378100 in GenBank) using the BLAST Ring Image Generator (BRIG, version 0.95) (Alikhan et al., 2011). Putative ICE was identified by ICEberg $^{3}$ and then compared with previously reported ICEKps by Kleborate ${ }^{4}$.

\section{Phylogenetic Analysis}

Ridom SeqSphere + software (version 5.0, Ridom GmbH, Germany) was used for the core genome multi-locus

\footnotetext{
${ }^{1}$ https://bigsdb.pasteur.fr/klebsiella/klebsiella.html

${ }^{2}$ https://bigsdb.pasteur.fr/cgi-bin/bigsdb/bigsdb.pl?db=pubmlst_klebsiella_seqdef ${ }^{3} \mathrm{http} / / / \mathrm{db}-\mathrm{mml}$. sjtu.edu.cn/ICEberg/

${ }^{4}$ https://github.com/katholt/Kleborate
} 
sequence typing (cgMLST) analysis with the whole genome sequence. Compared with common typing method MLST of 7 housekeeping genes or pulse field gel electrophoresis (PFGE), cgMLST scheme shows higher discriminatory power (PérezLosada et al., 2018; Gona et al., 2020). cgMLST schemes consist of a fixed set of conserved genome-wide genes. Alleles are used instead of single nucleotide polymorphisms (SNP) or concatenated sequences to mitigate the effects of recombination and to enable for a global and public nomenclature. For K. pneumoniae, 2358 target genes named with allelic nomenclature are employed in a cgMLST scheme, with NTUH-K2044 as the reference genome ${ }^{5}$. Similarly, 2526 accessory target genes were also applied for accessory genome allelic analysis ${ }^{6}$. Neighbor-joining trees based on core- and accessory genome allelic profile were constructed and the corresponding pairwise distances were calculated.

\section{Hypermucoviscosity Phenotype Identification}

Hypermucoviscosity phenotype was determined by string test. As previously described, a string test result was determined to be positive when a viscous filament greater than $5 \mathrm{~mm}$ in length was generated by stretching a bacterial colony with a bacteriological inoculation loop on a blood agar plate (Hadano, 2013; Shon et al., 2013).

\section{Serum Resistance Assay}

Three independent cultures for each strain were grown overnight and diluted to 1:1000 in $\mathrm{MH}$ broth with or without 20\% normal human serum. Three replicates of each culture were aliquoted into a flat-bottom 100 -well plate. The plate was incubated at $37^{\circ} \mathrm{C}$ with agitation. The $\mathrm{OD}_{600}$ of each culture was recorded every

\footnotetext{
${ }^{5}$ https://www.cgmlst.org/ncs/schema/schema/2187931/

${ }^{6}$ https://www.cgmlst.org/ncs/schema/2192830/
}

5 min for 10 h by using the Bioscreen C Automated Microbiology Growth Curve Analysis System (Oy Growth Curves Ab Ltd., Turku, Finland). NUTH-K2044 was employed as hvKP reference strain, while American Type Culture Collection (ATCC) 700603 as the non-hvKP reference.

\section{Statistical Analysis}

Comparison between continuous variables (allelic gene difference) was performed by the Mann-Whitney test, as they were not normally distributed. In serum resistance assay, records for $\mathrm{OD}_{600}$ at each time point were summarized as mean plus $95 \%$ confidence interval.

\section{RESULTS}

\section{Characteristics of the Strain}

A 74-year-old man, with predisposed type II diabetes mellitus and schistosomiasis cirrhosis, presented with fever and painful swelling of left neck for 1 week before admission. Computed tomographic scan revealed an abscess $(66 \mathrm{~mm} \times 42 \mathrm{~mm})$ on the left side of neck (Figure 1A). During this hospitalization, both cultures from the neck abscess and blood yielded K. pneumoniae. The two strains were later determined as the same via whole genome sequencing. The patient was eventually discharged home in good condition.

The strain Kp_whw was resistant to ampicillin but susceptible to the majority of the other antimicrobial agents, including cephalosporins, quinolones and carbapenems (Table 1). KP_whw looked shiny and cream-colored on blood agar and showed hypermucoviscosity phenotype with positive string test (Figure 1B). The strain belonged to ST 1049 and KL 5. We compared the $w z i$ locus of this strain (wzi208) with other known loci associated with hypervirulent serotype (wzi1, wzi2, and wzi5) (Figure 2). The length of wzi208 sequence is $447 \mathrm{bp}$ and there
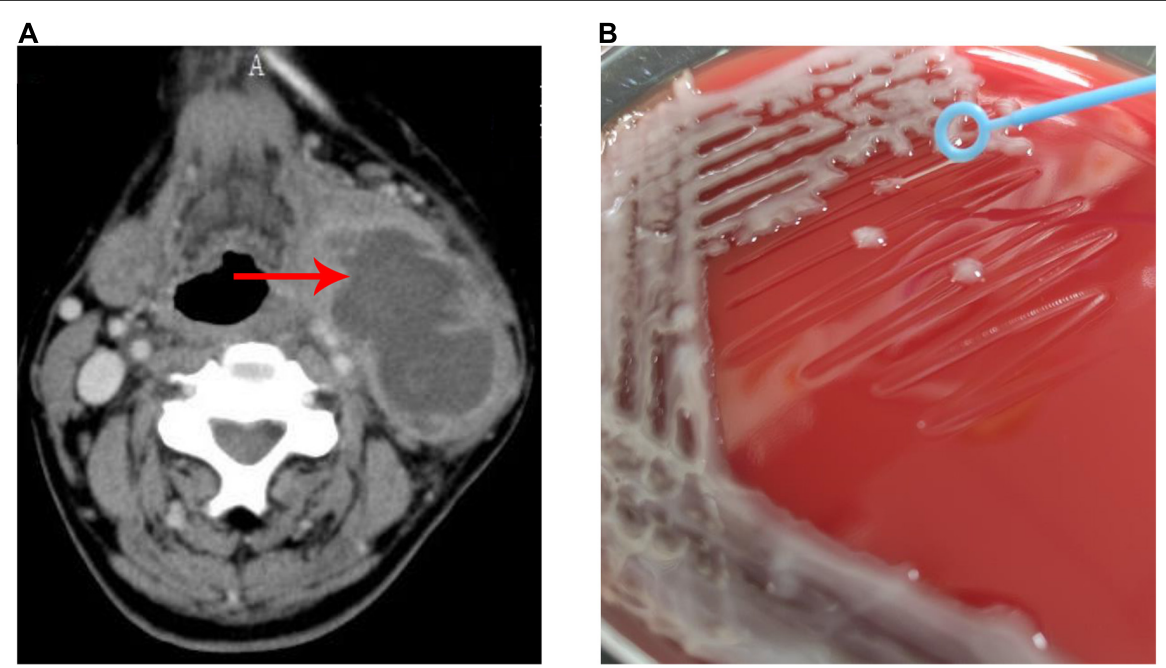

FIGURE 1 | Neck abscess caused by K. pneumoniae strain Kp_whw. (A) Computer tomography image showed an abscess (66 $\times 42$ mm) on the left side of neck. (B) String test for the strain Kp_whw. 
TABLE 1 | Susceptibility of Kp_whw to antimicrobial agents.

\begin{tabular}{lcc}
\hline Antimicrobial agent & MIC $(\boldsymbol{\mu} \mathbf{g} / \mathbf{m l})$ & Interpretation \\
\hline Ampicillin & 16 & $\mathrm{R}$ \\
Piperacillin & $\leq 4$ & $\mathrm{~S}$ \\
Amoxicillin/Clavulanic acid & $\leq 4 / 2$ & $\mathrm{~S}$ \\
Ampicillin/Sulbactam & $\leq 4 / 2$ & $\mathrm{~S}$ \\
Piperacillin/Tazobactam & $\leq 4 / 4$ & $\mathrm{~S}$ \\
Ceftazidime & $\leq 1$ & $\mathrm{~S}$ \\
Cefotaxime & $\leq 1$ & $\mathrm{~S}$ \\
Cefepime & $\leq 2$ & $\mathrm{~S}$ \\
Aztreonam & $\leq 2$ & $\mathrm{~S}$ \\
Imipenem & $\leq 1$ & $\mathrm{~S}$ \\
Amikacin & $\leq 8$ & $\mathrm{~S}$ \\
Gentamycin & $\leq 2$ & $\mathrm{~S}$ \\
Ciprofloxacin & $\leq 0.5$ & $\mathrm{~S}$ \\
Levofloxacin & $\leq 1$ & $\mathrm{~S}$ \\
Sulfamethoxazole-trimethoprim & $\leq 0.5 / 9.5$ & $\mathrm{~S}$ \\
Chloramphenicol & $\leq 4$ & $\mathrm{~S}$ \\
Tetracycline & $\leq 2$ & $\mathrm{~S}$ \\
Cefperazone/Sulbactam & $\leq 8$ & $\mathrm{~S}$ \\
Cefuroxime & $\leq 8$ & $\mathrm{~S}$ \\
Meropenem & $\leq 1$ & $\mathrm{~S}$ \\
\hline MiC minimum inhibitory concentration: $R$, &
\end{tabular}

MIC, minimum inhibitory concentration; $R$, resistant; $S$, susceptible.

were 31 SNPs difference in comparison with wzil and 24 SNPs with wzi2. However, wzi208 is closest to wzi5 (associated with K5 type), with only one SNP (C314A) (Figure 2).

\section{hvKP of K1/K2 Serotype}

The genetic similarity of strain Kp_whw with other K1/K2 serotype hvKP strains was analyzed. A total of 94 hvKP strains of $\mathrm{K} 1 / \mathrm{K} 2$ serotype (K1, $n=64 ; \mathrm{K} 2, n=30$ ) were included. Of the $95 \mathrm{~K}$. pneumoniae strains including Kp_whw, more than half of the strains were isolated from liver abscess $(65 / 95$, $67.4 \%$ ) and 13 (13.7\%) were from sputum (Figure 3A). Of note, the two isolated from bloodstream were strain NTUHK2044 (ST23, K1 serotype, causing liver abscess and metastatic meningitis) (Wu et al., 2009) and strain hvKP1 (causing liver abscess with metastatic spread to the spleen, ST86, K2 serotype) (Russo and Gill, 2013). Similarly, Kp_whw caused invasive infections i.e., neck abscess and bloodstream infection. Minimum spanning tree (MST) based on MLST showed the relationship between strain Kp_whw and other K1/K2 serotype hvKP (Figure 3B). ST23, ST65, and ST86 were the top three clones of hvKP. Compared with ST374 (K2), strain Kp_whw had five variant loci.

\section{Virulence Profile}

Most K1 serotype strains were ST23 (56/64, 87.5\%), while those K2 serotype showed more diversity, with 11 ST65 (36.7\%), 10 ST86 (33.3\%), 3 ST375 (10.0\%), 3 ST380 (10.0\%), 2 ST25 (6.7\%), and 1 ST374 (3.3\%) (Figure 4). Mucoviscosity-associated gene A (magA), which contributes largely to invasive infection but is specific to K1 serotype (Fang et al., 2004; Chuang et al., 2006), was not detected in Kp_whw. However, both regulator of mucoid phenotype $\mathrm{A}(r m p A)$ and $r m p A 2$ genes were present in Kp_whw. All the K2 type strains and Kp_whw showed positive string test, while less than a half of K1 type strain $(46.9 \%, 30 / 64)$ exhibited hypermucoviscosity phenotype.

\section{Mobile Genetic Elements Carrying Virulence Determinants}

Virulence plasmid pLVPK was well characterized previously (Chen et al., 2004). Alignment of strain Kp_whw contigs to pLVPK showed that Kp_whw carried a plasmid that aligned well to most parts of the PLVPK plasmid, including the region in which $r m p A, \operatorname{rmp} A 2$, and $\operatorname{iroBCDN}$ (encoding salmochelin system) and iucABCDiutA (encoding aerobactin system) genes were located, as well as silver and tellurite resistance gene clusters (silABCRS and terABCDEXYZW) (Figure 5A). In addition, a chromosomal ICEKp5-like mobile genetic element was detected in the strain. A $y b t$ locus encoding the biosynthesis of the siderophore yersiniabactin and its receptor was mobilized by this element (Figure 5B). These findings indicated that the strain Kp_whw harbored rich virulence factors and had the genetic potential to exacerbate the dissemination of hypervirulence.

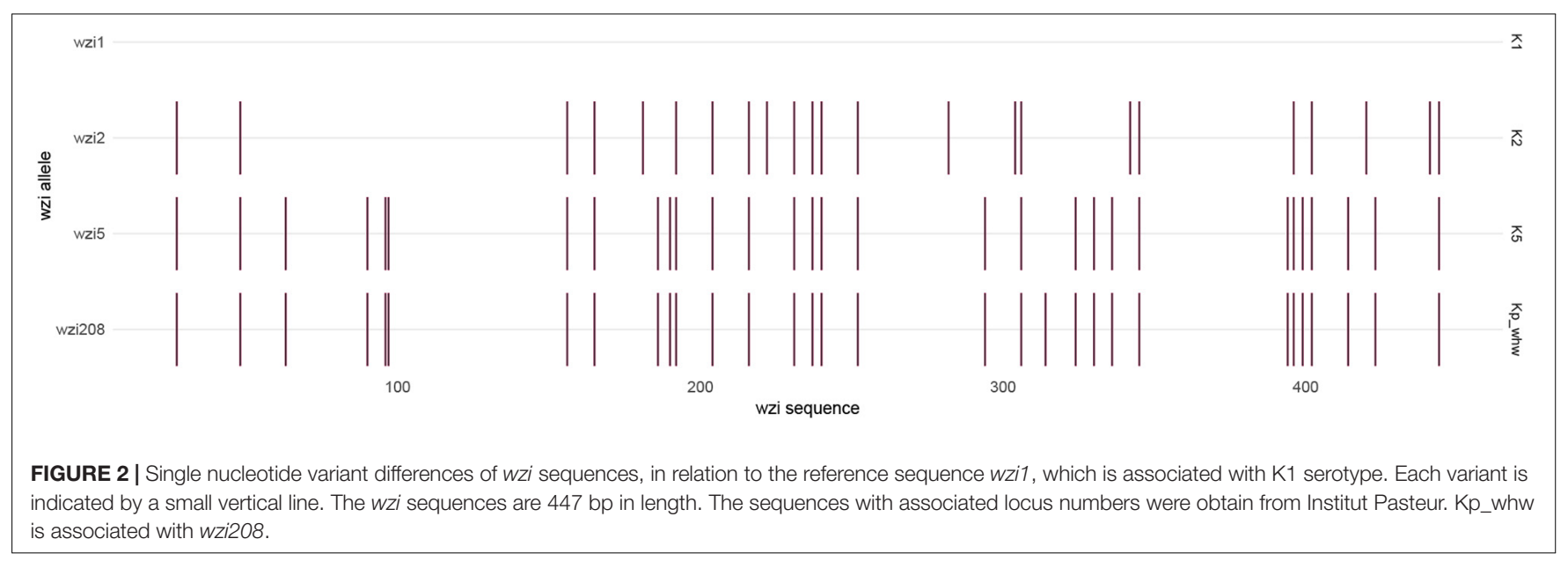




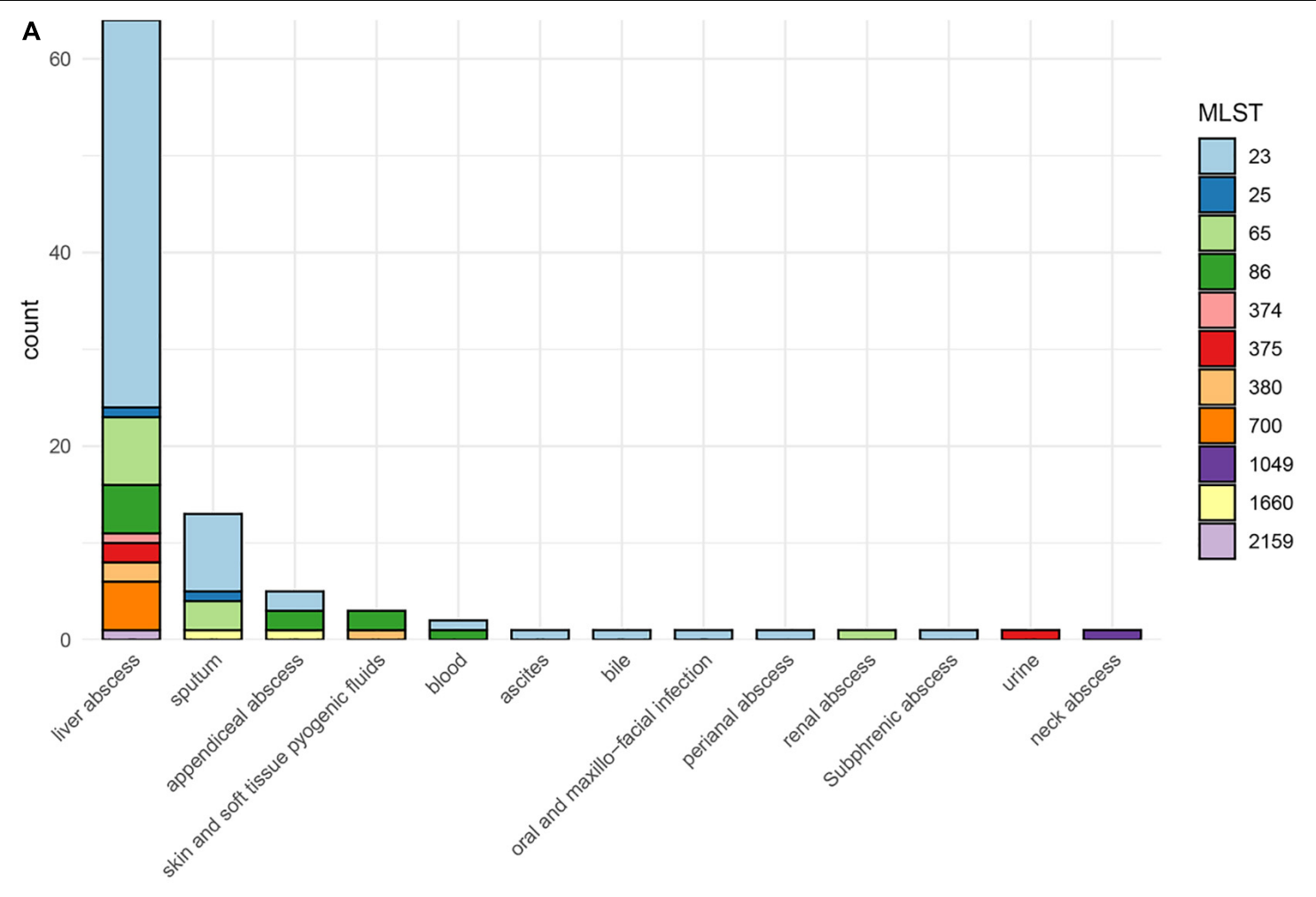

B

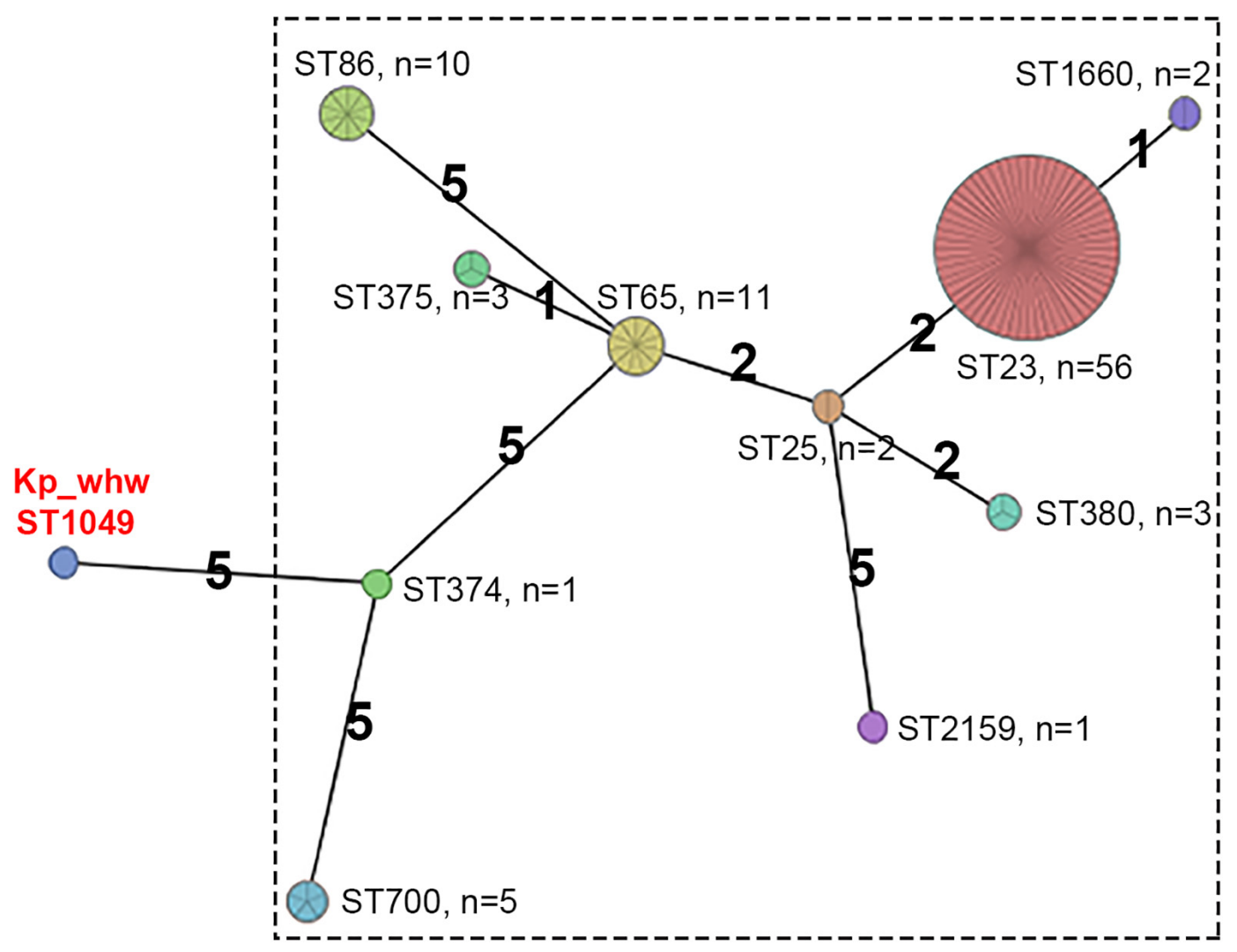

FIGURE 3 | Additional 94 K1/K2 serotype hvKP strains compared with Kp_whw. (A) Distribution of specimen sources of the 95 strains (including Kp_whw) according to sequence type. (B) Minimum spanning tree (MST) of the 95 strains based on multilocus sequence typing (MLST). 


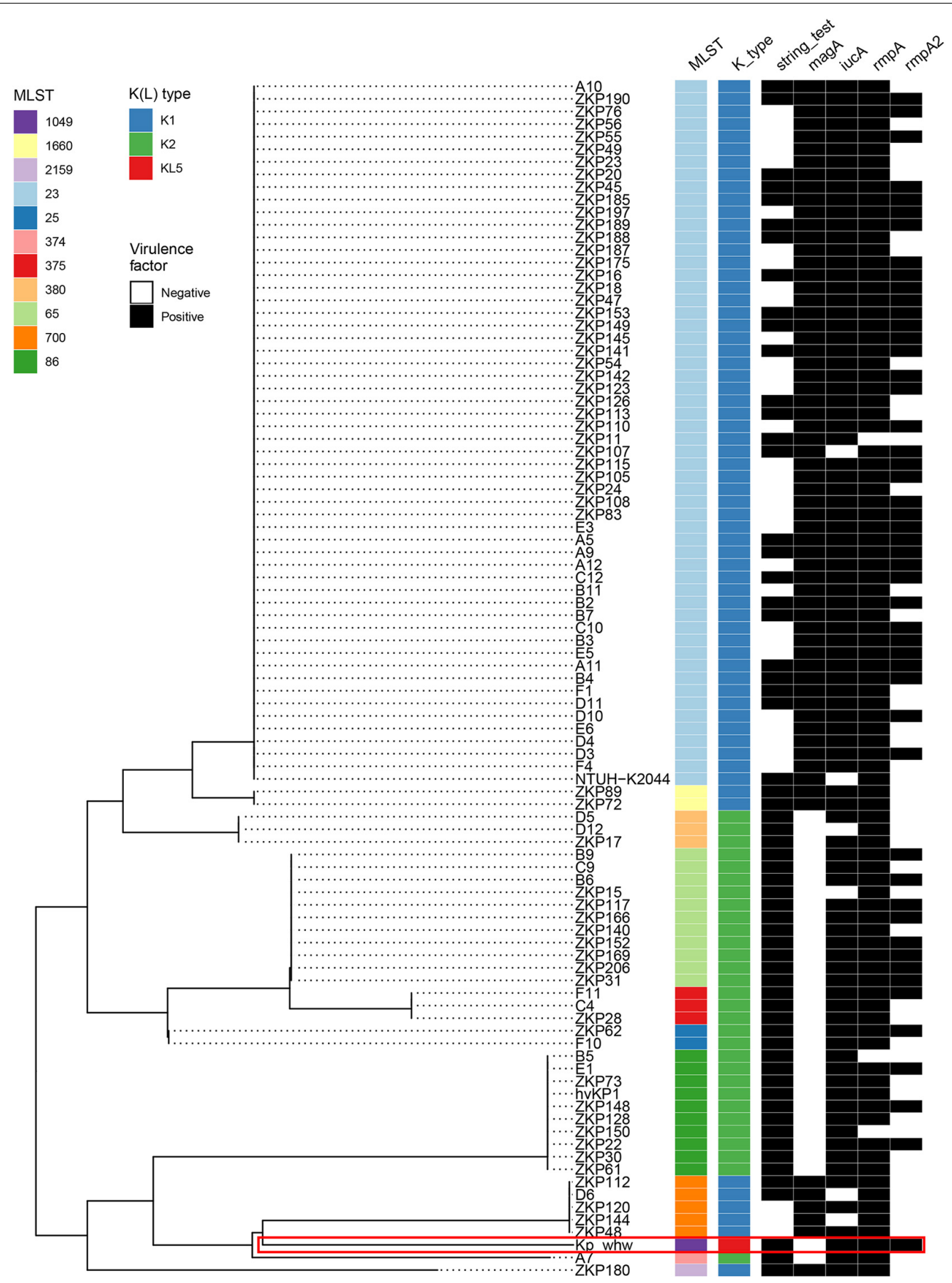

FIGURE 4 | Virulence profile of the 95 hvKP strains including Kp_whw. A MLST-based phylogenetic tree was constructed for all strains. ST and K(L) type were colored according to inset legend. The presence of the virulence factors including positive string test, magA gene, iucA gene, $r m p A$, and $r m p A 2$ genes were indicated as black rectangles. The position of Kp_whw was indicated with a red box.

\section{Comparative Analysis and Phylogenetic Trees}

The phylogenetic tree based on accessory gene allelic profile reflected almost completely the phylogeny of the core gene alleles (Figure 6A). One notable exception to this trend was identified in Kp_whw (ST1049), which was closed to strain ZKP180 (ST2159,
K1 serotype) based on accessory gene profile but unexpectedly formed an individual branch according to core gene alleles (Figure 6A). This was in accordance with the finding that allelic differences between the strain Kp_whw and K1/K2 serotype hvKP strains based on core gene profile were significantly larger than those from accessory gene profile (Figure 6B). 


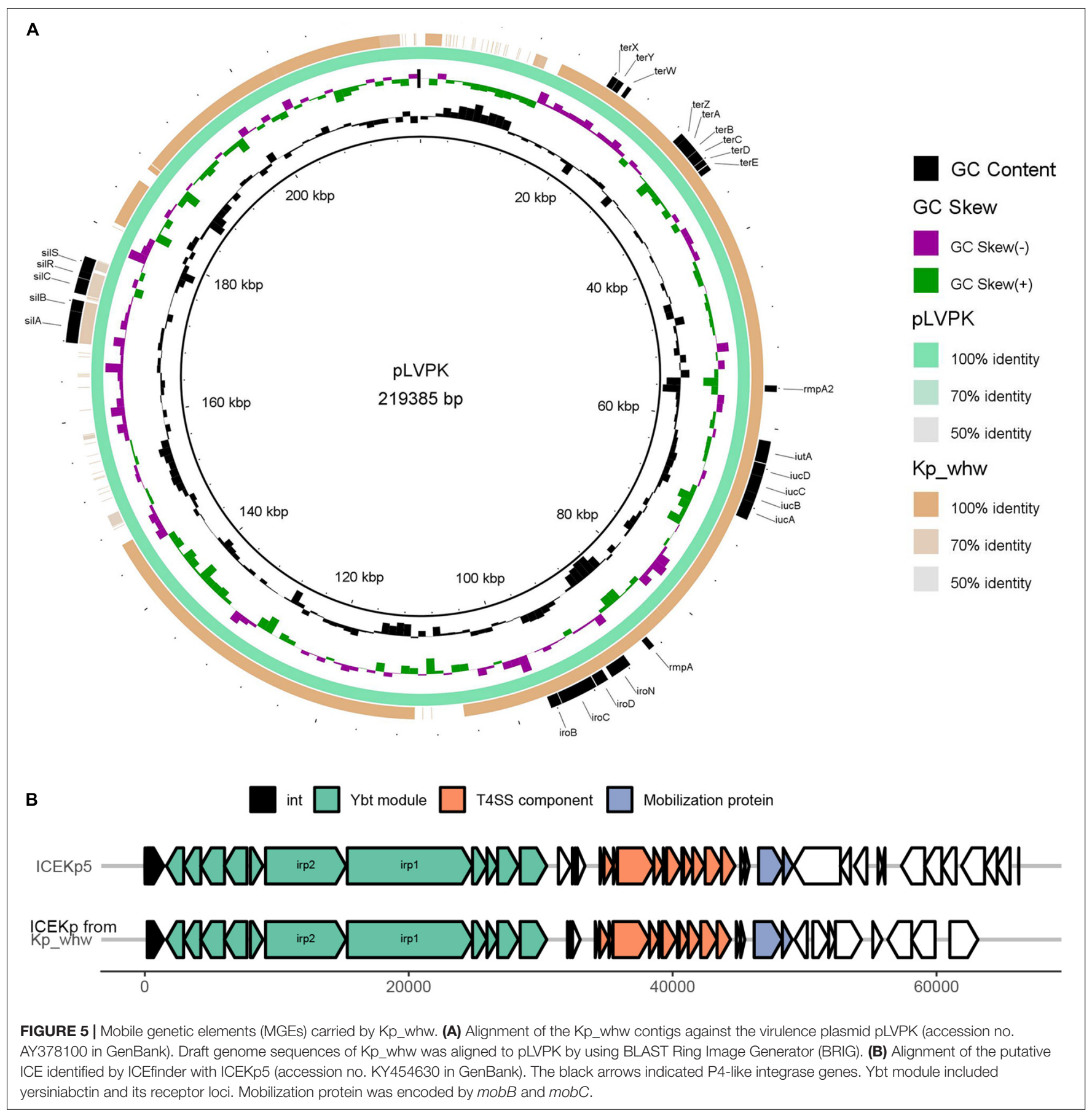

\section{Virulence Assessment}

To determine the virulence level of Kp_whw, serum resistant assay was performed. In $\mathrm{MH}$ broth without any supplement, ATCC 700603 outgrew NTUH-K2044 and Kp_whw (Figure 7). While in MH media with $20 \%$ normal human serum, however, Kp_whw and NTUH-K2044 were seldom influenced, yet ATCC700603 was dramatically inhibited to grow (Figure 7). Kp_whw and NTUH-K2044 had similar resistance to human serum, indicating that Kp_whw was as virulent as NTUH-K2044 in vitro.

\section{DISCUSSION}

Hypervirulent $K$. pneumoniae raised great concern recently. Here, we described a hvKP strain of ST1049, which was rarely reported, causing neck abscess, and invasive infection. hvKP is usually characterized as hypermucoviscosity, positive for $r m p A$, aerobactin, or virulence plasmid, and these features are frequently used to define hvKP. However, no single feature could accurately distinguish hvKP from non-hvKP (Lan et al., 2020). Clinical definition of hvKP based on the invasive liver abscess syndrome 

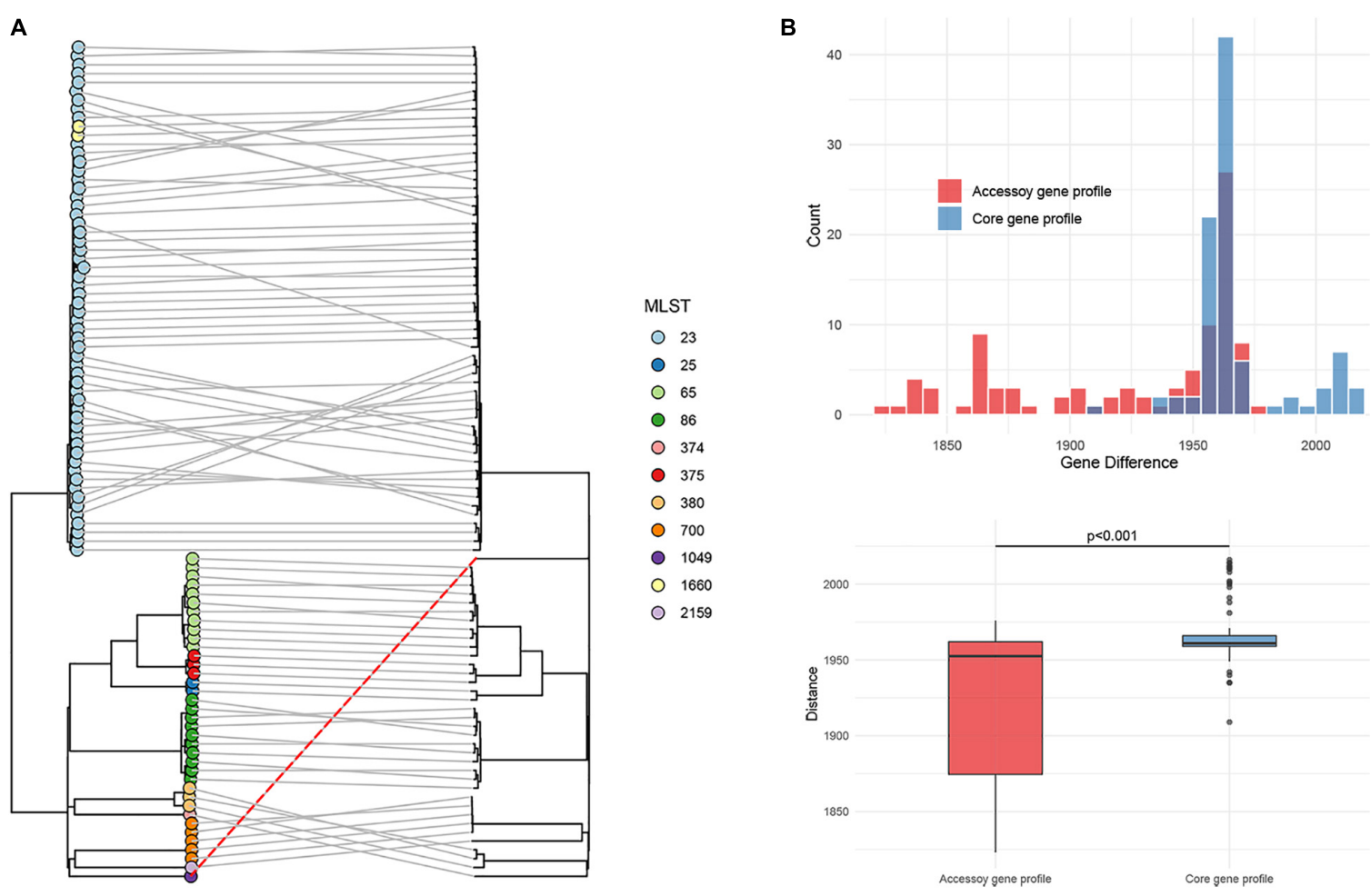

FIGURE 6 | Phylogenetic analysis of the 95 hvKP strains. (A) The phylogeny based on accessory gene allelic profile (left) was compared with the phylogeny based on the core genomic allelic profile. Kp_whw was indiacted with a dashed red line. Sequence types were colored according to inset legend. (B) Allelic gene differences on accessory genomic level between Kp_whw and K1/K2 strains were compared with those of core genomic level. Allelic gene differences were calculated by Ridom SeqSphere software.

and microbiological definition of $\mathrm{K} 1 / \mathrm{K} 2$ serotype have been accepted in recent years (Siu et al., 2012).

Neck infection is usually associated with dental procedure or oral infection (Walia et al., 2014). Although neck abscess caused by $K$. pneumoniae have been occasionally reported (Nadasy et al., 2007; Frazee et al., 2009), the genetic background and virulence level for the causative strains were seldom determined. A large retrospective analysis about deep neck abscess in China showed that $K$. pneumoniae was the most predominant gramnegative pathogen (Yang et al., 2008). Though more than a half neck abscess tended to be polymicrobial (Yang et al., 2008), most invasive soft tissue infection caused by $K$. pneumoniae were found to be monomicrobial (Park et al., 2019; Rahim et al., 2019), partly due to the survival advantages of hvKP, such as hypermucoviscosity and high siderophore production (Rahim et al., 2019). Owing to the invasiveness, Kp_whw cuased neck abscess and bloodstream infection, which was also reported with classical hvKP NTUH-K2044 (Wu et al., 2009) and hvKP1 (Russo and Gill, 2013). hvKP as a monomicrobial pathogen in invasive soft tissue infection is recently increasing and causes high morbidity and mortality (Rahim et al., 2019). Diabetes mellitus was frequently associated with $K$. pneumoniae infection (Cheng et al., 2015) and was considered as a critical risk factor (Sharma et al., 2018). The other two cases of neck abscess caused by hvKP in the United States were also associated with diabetes (Nadasy et al., 2007; Frazee et al., 2009).
This might be explained by the evidence that diabetic patients have defects on the neutrophil chemotactic and phagocytic activities (Alba-Loureiro et al., 2007). Of note, both the patients were Asia descent, and one had both neck abscess and blood positive culture for $K$. pneumoniae and the other developed metastatic complications.

Klebsiella pneumoniae of ST1049 was seldom reported globally. An epidemiological study on liver abscess caused by $K$. pneumoniae revealed that five cases were attributed to ST1049 K. pneumoniae (5/163, 3.1\%) (Zhang et al., 2019). However, microbiological features, virulence level, and genomic information of these strains were not analyzed. In the present study, we analyzed the K-locus for Kp_whw and determined that it belonged to KL5. The wzi208 (a region of K-locus) for Kp_whw is closed to wzi5, which is associated with K5 type. In Taiwan area of China, a ST1049 strain causing meningitis also belonged to $\mathrm{K} 5$ serotype (Ku et al., 2017). Actually, K. pneumoniae of K5 serotype is also a hypervirulent clone (Holt et al., 2015) and has frequently resulted in liver abscess (Lee et al., 2016), meningitis (Ku et al., 2017), and other invasive infections (Turton et al., 2010; Guo et al., 2017). From this perspective, Kp_whw possesses hypervirulent potential. On the other hand, Kp_whw was distant from K1/K2 serotype hvKP strains according to MLST and cgMLST. K. pneumoniae virulence factors are encoded by genes in both the core and accessory genomes (Martin and Bachman, 2018). LPS, siderophore enterobactin and 


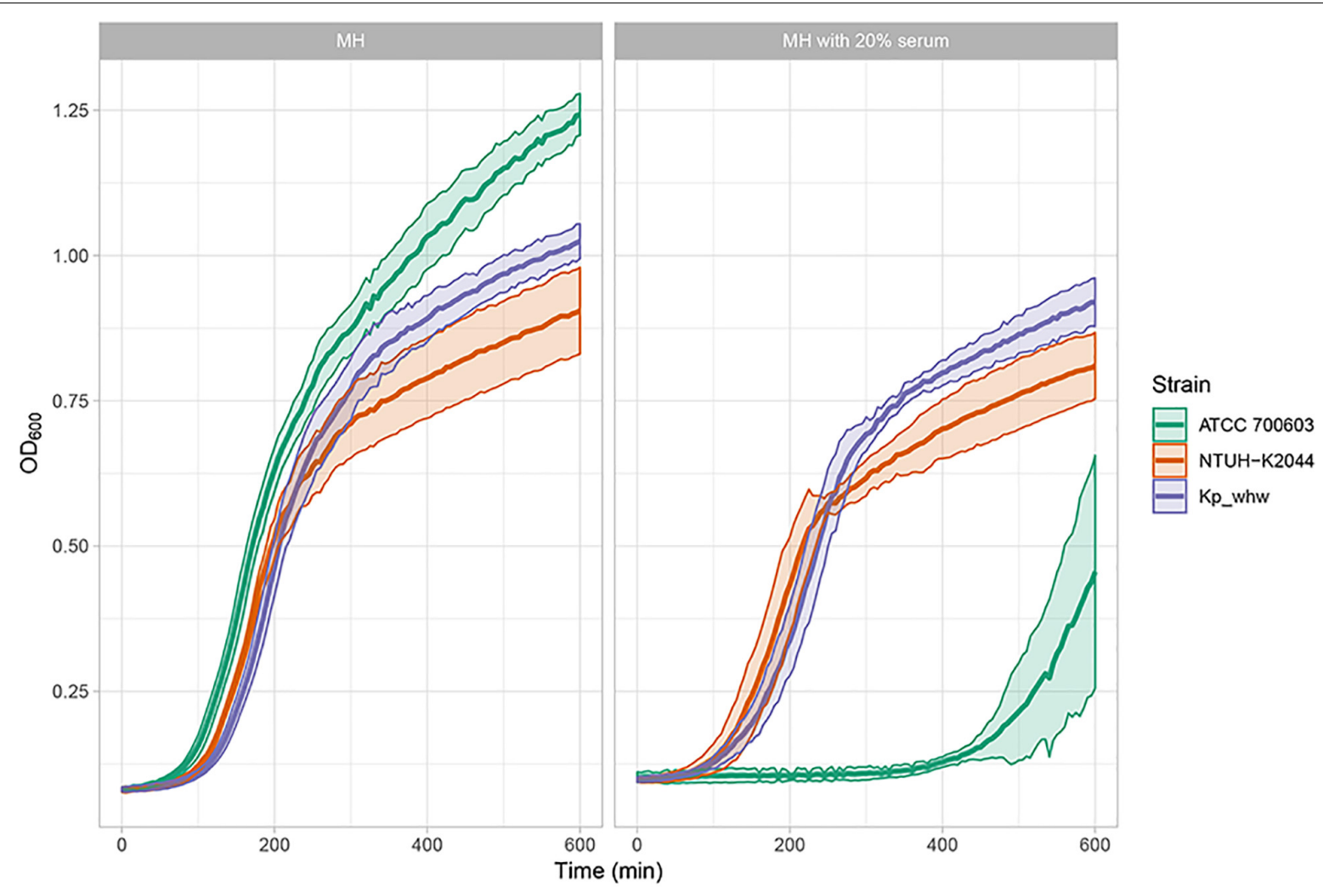

FIGURE 7 | Virulence level assessment in vitro. As microorganisms grow, they increase the turbidity of their growth medium. By measuring the turbidity of the medium over time, an $\mathrm{OD}_{600}$ curve can be generated. The curve reflects the growth (increased concentration) of the organism.

polysaccharide capsule synthesis are relative conserved and are primarily encoded by core genes while siderophore salmochelin, yersiniabactin and aerobactin are frequently ICE- or plasmidencoded (Martin and Bachman, 2018). Compared with K1/K2 type hvKP, Kp_whw presented similar accessory genomic profile, with relative diverse allelic variation of conversed genes. These indicated that the relative higher level of core genome allelic diversity might determine the differences of genetic background between Kp_whw and hvKP strains of K1/K2 serotype.

Plasmids and ICEs are two key components contributing to HGT (Wozniak and Waldor, 2010). ICEKp is one of selftransmissible mobile genetic elements (MGEs) which encode the machinery for conjugation (type IV secretion system, T4SS) and intricate regulatory systems to control their excision from the chromosome in the host (Wozniak and Waldor, 2010). ICEKp mainly mobilizes $y b t$ locus, which was first reported on the Yersinia high pathogenicity island (HPI), encoding yersiniabactin and its receptor. Yersiniabactin promoted the progression of bubonic and pneumonic plague, by scavenging iron directly from transferrin and lactoferrin (Fetherston et al., 2010). In vivo, yersiniabactin was demonstrated more important for K. pneumoniae growth than enterobactin under iron-limited conditions (Lawlor et al., 2007) and could facilitate microbes to colonize in the lungs and to cause disseminated infection (Lawlor et al., 2007; Bachman et al., 2011). Kp_whw carried an ICEKp5-like element, which containing a ybt14 locus, potentiating the transfer of yersiniabactin. Actually, additional siderophore like salmochelin (encoded by iro locus) can also be regulated by ICEKp (such as ICEKp1) (Lin et al., 2008). However, salmochelin, as well as aerobactin (encoded by iuc locus), are more frequently encoded by virulence plasmid, such as pLVPK (Chen et al., 2004). Though the affinity of aerobactin for iron is much lower than enterobactin (Brock et al., 1991), very low aerobactin concentrations are sufficient to stimulate bacterial growth (Garenaux et al., 2011), and it is essential for hvKP infection (Russo et al., 2015). For Kp_whw strain, iuc locus, iro locus and $r m p A / r m p A 2$ are simultaneously located in a single plasmid, which makes one concern about the acquisition of this type of plasmid by multidrug resistant strains.

For a long time, virulence plasmid was deemed congenital. Carbapenem-resistant (CR) hvKP strains are increasingly emerging recently, with known mechanism of the acquisition of antimicrobial resistance genes by hvKP, but not the acquisition of virulence plasmid by CRKP, due to the nonconjugative nature of virulence plasmid (Yang et al., 2019). However, CRKP strains gaining a virulence plasmid have recently caused a lethal outbreak in China (Gu et al., 2018). It is particularly worrying that a newly reported virulence plasmid p15WZ-82_Vir could be conjugated to CRKP strains, leading to the expression of carbapenem resistanceand hypervirulence-associated phenotypes simultaneously 
(Yang et al., 2019). Thus, it is now our urgency to pay attention to the convergence of hypervirulence and multi-drug resistance.

In conclusion, we characterized the genetic background and virulence level of a ST1049 K. pneumoniae strain Kp_whw isolated from neck abscess. Kp_whw is susceptible to commonly used antimicrobial agents except for being resistant to ampicillin. Kp_whw carried a pLVPK-like virulence plasmid and harbored a ICEKp5-like mobile genetic element. All results make clear that Kp_whw is a hvKP strain and has the potential to disseminate the phenotype, which poses a substantial public health threat. Although this is the first work characterizing the genetic virulence determinants of a ST1049 hvKP strain, only one strain was analyzed. In the future, isolates with diverse clinical characteristics should be collected for deep analysis.

\section{DATA AVAILABILITY STATEMENT}

The datasets presented in this study can be found in online repositories. The names of the repository/repositories and

\section{REFERENCES}

Alba-Loureiro, T. C., Munhoz, C. D., Martins, J. O., Cerchiaro, G. A., Scavone, C., Curi, R., et al. (2007). Neutrophil function and metabolism in individuals with diabetes mellitus. Braz. J. Med. Biol. Res. 40, 1037-1044. doi: 10.1590/s0100879x2006005000143

Alikhan, N. F., Petty, N. K., Ben Zakour, N. L., and Beatson, S. A. (2011). BLAST ring image generator (BRIG): simple prokaryote genome comparisons. BMC Genom. 12:402. doi: 10.1186/1471-2164-12-402

Bachman, M. A., Oyler, J. E., Burns, S. H., Caza, M., Lepine, F., Dozois, C. M., et al. (2011). Klebsiella pneumoniae yersiniabactin promotes respiratory tract infection through evasion of lipocalin 2. Infect. Immun. 79, 3309-3316. doi: 10.1128/IAI.05114-11

Brock, J. H., Williams, P. H., Liceaga, J., and Wooldridge, K. G. (1991). Relative availability of transferrin-bound iron and cell-derived iron to aerobactinproducing and enterochelin-producing strains of Escherichia coli and to other microorganisms. Infect. Immun. 59, 3185-3190. doi: 10.1128/iai.59.9.31853190.1991

Catalan-Najera, J. C., Garza-Ramos, U., and Barrios-Camacho, H. (2017). Hypervirulence and hypermucoviscosity: two different but complementary Klebsiella spp. phenotypes? Virulence 8, 1111-1123. doi: 10.1080/21505594. 2017.1317412

Chen, Y. T., Chang, H. Y., Lai, Y. C., Pan, C. C., Tsai, S. F., and Peng, H. L. (2004). Sequencing and analysis of the large virulence plasmid pLVPK of Klebsiella pneumoniae CG43. Gene 337, 189-198. doi: 10.1016/j.gene.2004.05.008

Cheng, N. C., Tai, H. C., Chang, S. C., Chang, C. H., and Lai, H. S. (2015). Necrotizing fasciitis in patients with diabetes mellitus: clinical characteristics and risk factors for mortality. BMC Infect. Dis. 15:417. doi: 10.1186/s12879015-1144-0

Chou, A., Nuila, R. E., Franco, L. M., Stager, C. E., Atmar, R. L., and Zechiedrich, L. (2016). Prevalence of hypervirulent Klebsiella pneumoniae-associated genes rmpA and magA in two tertiary hospitals in Houston, TX, USA. J. Med. Microbiol. 65, 1047-1048. doi: 10.1099/jmm.0.000309

Chuang, Y. P., Fang, C. T., Lai, S. Y., Chang, S. C., and Wang, J. T. (2006). Genetic determinants of capsular serotype K1 of Klebsiella pneumoniae causing primary pyogenic liver abscess. J. Infect. Dis. 193, 645-654. doi: 10.1086/499968

Fang, C. T., Chuang, Y. P., Shun, C. T., Chang, S. C., and Wang, J. T. (2004). A novel virulence gene in Klebsiella pneumoniae strains causing primary liver abscess and septic metastatic complications. J. Exp. Med. 199, 697-705. doi: 10.1084/jem.20030857

Fetherston, J. D., Kirillina, O., Bobrov, A. G., Paulley, J. T., and Perry, R. D. (2010). The yersiniabactin transport system is critical for the pathogenesis of bubonic accession number(s) can be found below: https://www.ebi.ac.uk/ ena, PRJEB38367 and https://www.ebi.ac.uk/ena, PRJEB34922.

\section{AUTHOR CONTRIBUTIONS}

PL and DZ conceived the idea and designed the experiments. JG isolated the strain. PL and RY performed the experiments. QS analyzed the data. YJ and JZ helped with materials and reagents. PL wrote the manuscript. YY reviewed the manuscript. All authors have read and agreed to the published version of the manuscript.

\section{FUNDING}

This work was supported by the National Natural Science Foundation of China under Grant Nos. 81672067 and 81830069; and the Natural Science Foundation of Zhejiang Province, China under Grant No. LY17H190004.

and pneumonic plague. Infect. Immun. 78, 2045-2052. doi: 10.1128/iai.01 236-09

Frazee, B. W., Hansen, S., and Lambert, L. (2009). Invasive infection with hypermucoviscous Klebsiella pneumoniae: multiple cases presenting to a single emergency department in the United States. Ann. Emerg. Med. 53, 639-642. doi: 10.1016/j.annemergmed.2008.11.007

Garenaux, A., Caza, M., and Dozois, C. M. (2011). The Ins and outs of siderophore mediated iron uptake by extra-intestinal pathogenic Escherichia coli. Vet. Microbiol. 153, 89-98. doi: 10.1016/j.vetmic.2011.05.023

Gona, F., Comandatore, F., Battaglia, S., Piazza, A., Trovato, A., Lorenzin, G., et al. (2020). Comparison of core-genome MLST, coreSNP and PFGE methods for Klebsiella pneumoniae cluster analysis. Microb. Genom. 6:347. doi: 10.1099/ mgen.0.000347

Gu, D., Dong, N., Zheng, Z., Lin, D., Huang, M., Wang, L., et al. (2018). A fatal outbreak of ST11 carbapenem-resistant hypervirulent Klebsiella pneumoniae in a Chinese hospital: a molecular epidemiological study. Lancet Infect. Dis. 18, 37-46. doi: 10.1016/S1473-3099(17)30489-9

Guo, Y., Wang, S., Zhan, L., Jin, Y., Duan, J., Hao, Z., et al. (2017). Microbiological and clinical characteristics of hypermucoviscous Klebsiella pneumoniae isolates associated with invasive infections in China. Front. Cell. Infect. Microbiol. 7:24. doi: 10.3389/fcimb.2017.00024

Hadano, Y. (2013). String test. BMJ Case Rep. 2013:e008328. doi: 10.1136/bcr2012-008328

Hodgson, K., Morris, J., Bridson, T., Govan, B., Rush, C., and Ketheesan, N. (2015). Immunological mechanisms contributing to the double burden of diabetes and intracellular bacterial infections. Immunology 144, 171-185. doi: 10.1111/imm. 12394

Holt, K. E., Wertheim, H., Zadoks, R. N., Baker, S., Whitehouse, C. A., Dance, D., et al. (2015). Genomic analysis of diversity, population structure, virulence, and antimicrobial resistance in Klebsiella pneumoniae, an urgent threat to public health. Proc. Natl. Acad. Sci. U.S.A. 112, E3574-E3581. doi: 10.1073/pnas. 1501049112

Joob, B., and Wiwanitkit, V. (2017). Klebsiella pneumoniae invasive liver abscess syndrome and Endophthalmitis. J. Emerg. Med. 53:917. doi: 10.1016/j. jemermed.2017.03.050

Ku, Y. H., Chuang, Y. C., Chen, C. C., Lee, M. F., Yang, Y. C., Tang, H. J., et al. (2017). Klebsiella pneumoniae isolates from meningitis: epidemiology, virulence and antibiotic resistance. Sci. Rep. 7:6634. doi: 10.1038/s41598-017-06878-6

Lam, M. M. C., Wick, R. R., Wyres, K. L., Gorrie, C. L., Judd, L. M., Jenney, A. W. J., et al. (2018). Genetic diversity, mobilisation and spread of the yersiniabactinencoding mobile element ICEKp in Klebsiella pneumoniae populations. Microb. Genom. 4:e000196. doi: 10.1099/mgen.0.000196 
Lan, P., Shi, Q., Zhang, P., Chen, Y., Yan, R., Hua, X., et al. (2020). Core genome allelic profiles of clinical Klebsiella pneumoniae strains using a random forest algorithm based on multilocus sequence typing scheme for hypervirulence analysis. J. Infect. Dis. 221(Suppl._2), S263-S271. doi: 10.1093/infdis/jiz562

Lawlor, M. S., O'Connor, C., and Miller, V. L. (2007). Yersiniabactin is a virulence factor for Klebsiella pneumoniae during pulmonary infection. Infect. Immun. 75, 1463-1472. doi: 10.1128/IAI.00372-06

Lee, I. R., Molton, J. S., Wyres, K. L., Gorrie, C., Wong, J., Hoh, C. H., et al. (2016). Differential host susceptibility and bacterial virulence factors driving Klebsiella liver abscess in an ethnically diverse population. Sci. Rep. 6:29316. doi: $10.1038 /$ srep29316

Lin, J. C., Chang, F. Y., Fung, C. P., Yeh, K. M., Chen, C. T., Tsai, Y. K., et al. (2010). Do neutrophils play a role in establishing liver abscesses and distant metastases caused by Klebsiella pneumoniae? PLoS One 5:e15005. doi: 10.1371/ journal.pone.0015005

Lin, Y. T., Jeng, Y. Y., Chen, T. L., and Fung, C. P. (2010). Bacteremic communityacquired pneumonia due to Klebsiella pneumoniae: clinical and microbiological characteristics in Taiwan, 2001-2008. BMC Infect. Dis. 10:307. doi: 10.1186/ 1471-2334-10-307

Lin, J. C., Siu, L. K., Fung, C. P., Tsou, H. H., Wang, J. J., Chen, C. T., et al. (2006). Impaired phagocytosis of capsular serotypes K1 or K2 Klebsiella pneumoniae in type 2 diabetes mellitus patients with poor glycemic control. J. Clin. Endocrinol. Metab. 91, 3084-3087. doi: 10.1210/jc.2005-2749

Lin, T.-L., Lee, C.-Z., Hsieh, P.-F., Tsai, S.-F., and Wang, J.-T. (2008). Characterization of integrative and conjugative element ICEKpl-associated genomic heterogeneity in a Klebsiella pneumoniae strain isolated from a primary liver abscess. J. Bacteriol. 190, 515-526. doi: 10.1128/JB.01219-07

Lin, Y. T., Siu, L. K., Lin, J. C., Chen, T. L., Tseng, C. P., Yeh, K. M., et al. (2012). Seroepidemiology of Klebsiella pneumoniae colonizing the intestinal tract of healthy Chinese and overseas Chinese adults in Asian countries. BMC Microbiol. 12:13. doi: 10.1186/1471-2180-12-13

Liu, Y. C., Cheng, D. L., and Lin, C. L. (1986). Klebsiella pneumoniae liver abscess associated with septic endophthalmitis. Arch. Intern. Med. 146, 1913-1916. doi: 10.1001/archinte.146.10.1913

Martin, R. M., and Bachman, M. A. (2018). Colonization, infection, and the accessory genome of Klebsiella pneumoniae. Front. Cell. Infect. Microbiol. 8:4. doi: 10.3389/fcimb.2018.00004

Melot, B., Brisse, S., Breurec, S., Passet, V., Malpote, E., Lamaury, I., et al. (2016). Community-acquired meningitis caused by a CG86 hypervirulent Klebsiella pneumoniae strain: first case report in the Caribbean. BMC Infect. Dis. 16:736. doi: 10.1186/s12879-016-2065-2

Nadasy, K. A., Domiati-Saad, R., and Tribble, M. A. (2007). Invasive Klebsiella pneumoniae syndrome in North America. Clin. Infect. Dis. 45, e25-e28. doi: 10.1086/519424

Namikawa, H., Yamada, K., Sakiyama, A., Imoto, W., Yamairi, K., Shibata, W., et al. (2019). Clinical characteristics of bacteremia caused by hypermucoviscous Klebsiella pneumoniae at a tertiary hospital. Diagn. Microbiol. Infect. Dis. 95, 84-88. doi: 10.1016/j.diagmicrobio.2019.04.008

Paczosa, M. K., and Mecsas, J. (2016). Klebsiella pneumoniae: going on the offense with a strong defense. Microbiol. Mol. Biol. Rev. 80, 629-661. doi: 10.1128/ mmbr.00078-15

Park, S. Y., Yu, S. N., Lee, E. J., Kim, T., Jeon, M. H., Choo, E. J., et al. (2019). Monomicrobial gram-negative necrotizing fasciitis: an uncommon but fatal syndrome. Diagn. Microbiol. Infect. Dis. 94, 183-187. doi: 10.1016/j. diagmicrobio.2018.12.013

Peirano, G., Pitout, J. D., Laupland, K. B., Meatherall, B., and Gregson, D. B. (2013). Population-based surveillance for hypermucoviscosity Klebsiella pneumoniae causing community-acquired bacteremia in Calgary, Alberta. Can. J. Infect. Dis. Med. Microbiol. 24, e61-e64. doi: 10.1155/2013/828741

Pérez-Losada, M., Arenas, M., and Castro-Nallar, E. (2018). Microbial sequence typing in the genomic era. Infect. Genet. Evol. 63, 346-359. doi: 10.1016/j. meegid.2017.09.022

Prokesch, B. C., TeKippe, M., Kim, J., Raj, P., TeKippe, E. M., and Greenberg, D. E. (2016). Primary osteomyelitis caused by hypervirulent Klebsiella pneumoniae. Lancet Infect. Dis. 16, e190-e195. doi: 10.1016/s1473-3099(16)3 0021-4
Rafat, C., Messika, J., Barnaud, G., Dufour, N., Magdoud, F., Billard-Pomarès, T., et al. (2018). Hypervirulent Klebsiella pneumoniae, a 5-year study in a French ICU. J. Med. Microbiol. 67, 1083-1089. doi: 10.1099/jmm.0.000788

Rahim, G. R., Gupta, N., Maheshwari, P., and Singh, M. P. (2019). Monomicrobial Klebsiella pneumoniae necrotizing fasciitis: an emerging life-threatening entity. Clin. Microbiol. Infect. 25, 316-323. doi: 10.1016/j.cmi.2018.05.008

Russo, T. A., and Gill, S. R. (2013). Draft genome sequence of the hypervirulent Klebsiella pneumoniae strain hvKP1, isolated in Buffalo, New York. Genome Announc. 1:e0006513. doi: 10.1128/genomeA.00065-13

Russo, T. A., and Marr, C. M. (2019). Hypervirulent Klebsiella pneumoniae. Clin. Microbiol. Rev. 32:e0001-19. doi: 10.1128/cmr.00001-19

Russo, T. A., Olson, R., MacDonald, U., Beanan, J., and Davidson, B. A. (2015). Aerobactin, but not yersiniabactin, salmochelin, or enterobactin, enables the growth/survival of hypervirulent (hypermucoviscous) Klebsiella pneumoniae ex vivo and in vivo. Infect. Immun. 83, 3325-3333. doi: 10.1128/iai.00430-15

Sharma, K., Das, D., Joshi, M., Barman, D., and Sarma, A. J. (2018). Deep neck space infections-A study in diabetic population in a tertiary care centre. Indian J. Otolaryngol. Head. Neck Surg. 70, 22-27. doi: 10.1007/s12070-017-1196-0

Shon, A. S., Bajwa, R. P., and Russo, T. A. (2013). Hypervirulent (hypermucoviscous) Klebsiella pneumoniae: a new and dangerous breed. Virulence 4, 107-118. doi: 10.4161/viru.22718

Siu, L. K., Yeh, K. M., Lin, J. C., Fung, C. P., and Chang, F. Y. (2012). Klebsiella pneumoniae liver abscess: a new invasive syndrome. Lancet Infect. Dis. 12, 881-887. doi: 10.1016/s1473-3099(12)70205-0

Struve, C., Roe, C. C., Stegger, M., Stahlhut, S. G., Hansen, D. S., Engelthaler, D. M., et al. (2015). Mapping the evolution of hypervirulent Klebsiella pneumoniae. mBio 6:e00630. doi: 10.1128/mBio.00630-15

Turton, J. F., Perry, C., Elgohari, S., and Hampton, C. V. (2010). PCR characterization and typing of Klebsiella pneumoniae using capsular typespecific, variable number tandem repeat and virulence gene targets. J. Med. Microbiol. 59(Pt 5), 541-547. doi: 10.1099/jmm.0.015198-0

Walia, I. S., Borle, R. M., Mehendiratta, D., and Yadav, A. O. (2014). Microbiology and antibiotic sensitivity of head and neck space infections of odontogenic origin. J. Maxillofac. Oral. Surg. 13, 16-21. doi: 10.1007/s12663-012-0455-6

Wozniak, R. A. F., and Waldor, M. K. (2010). Integrative and conjugative elements: mosaic mobile genetic elements enabling dynamic lateral gene flow. Nat. Rev. Microbiol. 8, 552-563. doi: 10.1038/nrmicro2382

Wu, K. M., Li, L. H., Yan, J. J., Tsao, N., Liao, T. L., Tsai, H. C., et al. (2009). Genome sequencing and comparative analysis of Klebsiella pneumoniae NTUH-K2044, a strain causing liver abscess and meningitis. J. Bacteriol. 191, 4492-4501. doi: 10.1128/jb.00315-09

Wyres, K. L., Lam, M. M. C., and Holt, K. E. (2020). Population genomics of Klebsiella pneumoniae. Nat. Rev. Microbiol. 18, 344-359. doi: 10.1038/s41579019-0315-1

Yang, S. W., Lee, M. H., See, L. C., Huang, S. H., Chen, T. M., and Chen, T. A. (2008). Deep neck abscess: an analysis of microbial etiology and the effectiveness of antibiotics. Infect. Drug Resist. 1, 1-8. doi: 10.2147/idr.s3554

Yang, X., Wai-Chi Chan, E., Zhang, R., and Chen, S. (2019). A conjugative plasmid that augments virulence in Klebsiella pneumoniae. Nat. Microbiol. 4, 2039-2043. doi: 10.1038/s41564-019-0566-7

Zhang, S., Zhang, X., Wu, Q., Zheng, X., Dong, G., Fang, R., et al. (2019). Clinical, microbiological, and molecular epidemiological characteristics of Klebsiella pneumoniae-induced pyogenic liver abscess in southeastern China. Antimicrob. Resist. Infect. Control. 8:166. doi: 10.1186/s13756-019-0615-2

Conflict of Interest: The authors declare that the research was conducted in the absence of any commercial or financial relationships that could be construed as a potential conflict of interest.

Copyright (c) 2021 Lan, Zhao, Gu, Shi, Yan, Jiang, Zhou and Yu. This is an openaccess article distributed under the terms of the Creative Commons Attribution License (CC BY). The use, distribution or reproduction in other forums is permitted, provided the original author(s) and the copyright owner(s) are credited and that the original publication in this journal is cited, in accordance with accepted academic practice. No use, distribution or reproduction is permitted which does not comply with these terms. 